Aus Dr. Halles Poliklinik für Hals-, Nasen- und Ohrenleiden in Berlin.

\title{
Die Bedeutung der Myalgien für die oto-rhinologische Diagnostik.
}

Von Dr. Ernst Wodak, gew. Asssistent der deutschen Ohrenklinik in Prag.

Als ich im Jänner Ig2o zum ersten Male an der Halleschen Poliklinik eine große Reihe von Myalgiefällen sah, konnte ich mich, wie ich offen gestehe, einer gewissen Skepsis nicht erwehren, da es mir recht unwahrscheinlich schien, daß man derart verschiedene Krankheitsbilder, wie ich sie dort sah, unter den gemeinsamen Begriff der Myalgien bringen könne. Wenn man bedenkt, daß die Myalgien, vor allem des Sternocleidomastoideus und des Cucullaris, Symptome verursachen sollen, die auf Erkrankungen verschiedenartiger Organe (z. B. Ohr, Nasen-Nebenhöhlen, selbst Zerebrum usw.) hindeuten können, so wird man meine Skepsis verstehen.

Nach Hause zurückgekehrt, suchte ich mir an Hand meines eigenen Krankenmaterials ein Bild von den Myalgien zu machen und muß sagen, daß ich schon nach kurzer Zeit von dem Ergebnis meiner Beobachtungen überrascht war. Vor allem konnte ich bald feststellen, daß selbst unter meinem doch relativ kleinen Material Myalgien verhältnismäßig recht oft vorkamen. Dieser Umstand gab zu denken. Da die Fälle von Myalgien, auch bevor ich nach ihnen fahndete, sicher ebenso häufig waren, gehe ich mit der Annahme nicht fehl, daß ich sie oft einfach übersehen und nur in Fällen, wo direkt über rheumatische Beschwerden geklagt wurde, dem Internisten zugewiesen hatte. Nebst diesem ersten, etwas überraschenden Ergebnis konnte ich weiter die Erfahrung machen, daß ich durch sorgfältiges Forschen nach diesem Krankheitsbilde and seine planmäßige Behandlung nach Halles ( $I$ ) und Peritzs (2) Vorschriften oftmals in auffallend kurzer Zeit Heilung bei Fällen erzielen konnte, die vorher offenbar unter falscher Flagge gesegelt und ohne jeden Erfolg von anderer Seite behandelt worden waren. Diese erwähnten Beobachtungen häuften und wiederholten sich immer wieder, so daß sie in ihrer Gesamtheit $\mathrm{m}$. E. eine glänzende Bestätigung alles dessen darstellen, was Halle über das Krankheitsbild der Myalgien gesagt und geschrieben hatte. Schließlich wurde 
es mir zur Gewohnheit, in allen unklaren Fällen, in denen, wie Halle betont, der objektive Befund zu den intensiven Beschwerden in keinem Verhältnis steht, auf Myalgie zu untersuchen. Diese Gewohnheit hat mich, wie ich offen zugeben muß, sicherlich vor mancher Fehldiagnose bewahrt.

Alle diese eben geschilderten Erfahrungen machten andere Schüler Halles sowie Fachkollegen, die bei Halle Fälle von Myalgie gesehen hatten, nach ihren teils mündlichen, teils brieflichen Angaben im großen ganzen in gleicher Weise wie ich. Um so mehr muß es eigentlich Wunder nehmen, daß Halles warmer Appell vom Jahre IgII, I9I7 usw. wenigstens nach den Veröffentlichungen über dieses Thema zu schließen - recht wenig Widerhall gefunden hat. Man trifft nur hier und da ganz kurze Bemerkungen in der Literatur, die auf die Myalgien Bezug nehmen. So sagt z. B. Bárány (3) bei der Schilderung der Schmerzen am Warzenfortsatz in Fällen, die seinen Symptomenkomplex zeigen: „,Vor Verwechslung mit rheumatischen Schmerzen in den ansetzenden Muskeln hat man sich besonders zu hüten“. Bárány scheint also die Wichtigkeit der Myalgien an dieser Stelle wohl zu kennen. In jüngster Zeit weist auch Grahe (8) darauf hin.

Abgesehen von diesen wenigen verstreuten Bemerkungen finden wir in der Fachliteratur so gut wie nichts darüber. Und doch hätte dieses Krankheitsbild ganz besonderes Interesse verdient, da es für den in der Praxis stehenden Fachkollegen von großer Bedeutung ist.

Dabei ist es nicht allein unser Fach, für das der Symptomenkomplex der Myalgien eine große Rolle spielt, sondern die Durchsicht der Literatur zeigt, daß auch für andere Gebiete die Kenntnis dieser Affektion vonnöten ist. So wies bereits vor mehr als $30 \mathrm{Jahren}$ Rosen b a ch(4) darauf hin, daß Myalgien der Bauchmuskeln Herz- oder Magenleiden vortäuschen können. Er sagt darüber: „,Vor allem ist eine möglichst frühzeitige Diagnose von Wichtigkeit, damit man nicht, durch das Vorherrschen der Symptome gestörter Magentätigkeit irregeleitet, seine Maßnahmen gegen ein nicht vorhandenes Magenleiden richtet und das Magennervensystem noch empfindlicher macht". . Rosenbach erkennt auch - wohl als erster - den ursächlichen Zusammenhang zwischen Myalgie der großen Halsmuskeln und Migräne. Welche Beachtung dieser Hinweis Rosenbachs bei den Internisten gefunden hat, kann ich nicht beurteilen, doch besitzen seine Worte - mutatis mutandis - auch für unser Fach vollinhaltlich Gültigkeit.

Ich möchte mir nun im folgenden gestatten, einerseits an Hand kurzer, aber typischer Krankheitsfälle das mannigialtige Bild der Myalgien zu skizzieren, andererseits auch den Versuch einer Erklärung für diese auffallenden Zusammenhänge' zu machen. 
Die Symptome der Myalgien, soweit sie uns hier interessieren, lassen sich in drei Gruppen einteilen: I. In Allgemeinsymptome, 2. Symptome, die eine Ohrerkrankung und deren Komplikation vortäuschen können, 3. solche, die an eine Affektion der Nasen-Nebenhöhlen sowie deren Komplikation denken lassen.

I. Zur ersten Gruppe gehören alle jene Symptome, die wir auch sonst bei rheumatischen oder rheumatoiden Erkrankungen des übrigen Körpers finden, nur sind sie hier, da es sich um die zwei mächtigsten am Kopfe ansetzenden Muskeln handelt, entsprechend verändert. Das wichtigste Symptom ist der Kopfschmerz, der nicht nur an allen Teilen des Schädels, sondern auch in allen möglichen Qualitäten auftreten kann. Wir finden also Stirn-, Scheitel-, Schläfen- und Hinterkopfschmerzen, die ihrerseits wieder stechend, bohrend, dumpf usw. sein können. Doch kehrt meist das sog. "Kappengefühl" wieder. Die Patienten geben gewöhnlich an, früh das Gefühl zu haben, ,als ob der Kopf in einen Reifen gepreßt sei" (Rosenbach).

Von anderen Allgemeinsymptomen sei erwähnt: ein individuell verschieden entwickeltes, oft aber geradezu schweres Krankheitsgefühl, das in keiner Beziehung zum geringfügigen objektiven Befunde steht, ferner starker Schweißausbruch, Abgeschlagensein, Arbeitsunfähigkeit und schließlich 'Schlaflosigkeit, die aber nicht wie $z$. B. bei der GrippeEnzephalitis nervösen (zentralen) Ursprunges zu sein scheint. Die Myalgien der beiden großen Kopfmuskeln, des Sternocleidomastoideus und des Cucullaris, sowie der meist mitbeteiligten kurzen, tiefen Nackenmuskeln bewirken, daß jede Fixation des Kopfes, somit auch die kleinste aktive und passive Bewegung desselben, von intensivem, manchmal fast unerträglichem Schmerze begleitet ist. Dies kann sich gelegentlich so steigern, daß der Kopf wie in einer Schiene vollkommen starr gehalten wird und der Patient sich bemüht, nicht nur jede Bewegung des Kopfes, sondern auch jede Aktion der mimischen Gesichtsmuskulatur, ja, selbst der Schlundmuskeln, nach Möglichkeit zu vermeiden. Es ist begreiflich, daß in solchen Fällen sowohl Nahrungsaufnahme, als auch Allgemeinbefinden usw. gestört sind und es kann sogar infolge der Unmöglichkeit, im Bette eine geeignete, nicht schmerzhafte Lagerung für den Kopf einzunehmen, zu lange dauernder Schlaflosigkeit kommen. Hierfür konnte ich jüngst ein geradezu klassisches Beispiel in folgendem Falle sehen:

Der 33 jährige K. M. verspürte in der Gegend des Ansatzes des rechten Sternokleidomastoideus am Warzenfortsatze leichte Schmerzen, die nach einer starken Erkältung (4stündiger Aufenthalt im Regen) plötzlich an Intensität so zunahmen, daß er den Kopf nicht mehr bewegen konnte. Torticollis nach links. Patient konnte außerdem eine Woche lang aus oben näher erörterten Gründen nicht schlafen. Die 
einzige Art, wenigstens für ein paar Minuten in einen schlafähnlichen Zustand zu gelangen, war die, daß Patient sich in einen Stuhl setzte und den Kopf in beide Hände stützte; jede andere Lage war wegen unerträglicher Schmerzen unmöglich. - Aspirin in großen Mengen, heiße Umschläge, Heißluftbehandlung, Sțreichmassage durch ca. eine Woche blieben ohne jeden Erfolg. Erst Knetmassage nach Halle-Peritz führte in 3-4 Tagen rasch wesentliche Besserung herbei. - Hier waren der ïberaus schwere Allgemeinzustand, das Unvermögen, irgendwelche Bewegungen mit dem Kopfe oder der mimischen Muskulatur machen zu können, ja, selbst die hochgradige Erschwerung des Schluckaktes so auffallend, daß mị Patient von einem Internisten mit der Anfrage zugewiesen wurde, ob hier nicht eine Affektion des Pharynx bez. Larynx vorliege.

Ein ähnlicher, von Halle beobachteter und mir freundlichst zur Publikation überlassener Fall ist folgender:

Ein junges Mädchen klagt iiber heftige Schluckschmerzen, die schon seit Monaten von bekannten Fachkollegen vergeblich mit Pinselungen und Halsumschlägen behandelt wurden.

Im Pharynx und Larynx objektiv keine Entzündung nachweisbar, Mandeln o. B., keine Hyperästhesie noch irgendwelche nervöse Stigmata.

Die fortgesetzte Untersuchung ergab rechts sehr starke Myalgien, die -- unter Vermeidung jeder Suggestion - in geeigneter Weise massiert wurden. Nach 5 maliger Behandlung konnte Patientin schlucken. Wohlbefinden 'bei noch nicht ganz geschwundenen Muskelschmerzen nach 2 Wochen.

In anderen Fällen wieder können Myalgien Symptome hervorrufen, die - speziell bei Unkenntnis des Krankheitsbildes oder flüchtiger Untersuchung - gelegentlich den Eindruck einer zerebralen Affektion machen. So wurde ich vor kurzem zu einem ca. 40 jährigen Patienten gerufen, der in seinem Bureau plötzlich kollapsartig zusammengebrochen war und bei dem der herbeigerufene Arzt die Befürchtung einer zerebralen Erkrankung ausgesprochen hatte. Wie schwer der Zustand von den nächsten Angehörigen aufgefaßt wurde, erhellt daraus, da $\beta$ mir keim Betreten der Wohnung die Gattin des Patienten in Tränen aufgelöst und ganz gebrochen entgegen kam. Patient lag im Bette, hatte im Gesicht den Ausdruck eines schwer Leidenden und klagte über intensive Schmerzen im rechten Ohr sowie in der Scheitelgegend. Temperatur - wie meist bei Myalgien - annähernd normal, starker Schweißausbruch. Die objektive 'Untersuchung der Ohren sowie der oberen Luftwege usw, ergab völlig normalen Befund. Schon wollte ich Patienten einem Internisten zuweisen, als ich noch zuletzt gewohnheitsgemäß an den Cucullaris griff, worauf Patient vor Schmerz sich 
bäumte. Die Diagnose der Myalgie stand für mich sicher; Patient war auch nach 2 Tagen von seinen Hauptbeschwerden befreit.

2. Zur zweiten uns besonders interessierenden Gruppe sind jene Symptome zu zählen, bei denen der Facharzt versucht ist, sie auf eine Erkrankung des Ohres zu beziehen. Zu diesen Symptomen gehören vornehmlich Ohrensausen, Schwindelanfälle, Brechreiz, spontane und bei Druck auftretende Schmerzen am Warzenfortsatz und im Ohre selbst. Alle diese genannten Symptome gewinnen natürlich besonders an Bedeutung, wenn gleichzeitig eine eitrige - akute oder chronische Mittelohrentzündung besteht. Wenn es auch nicht angeht, aus den angeführten Krankheitserscheinungen allein eine Indikation zur Eröfnung des Warzenfortsatzes ableiten zu wollen, eine Aussicht, die sich mit den Anschauungen Brühls (5), Claus' (6) u. a. deckt, so läßt es sich doch nicht ganz von der Hand weisen, daß in zweifelhaften Fällen, in denen die Entscheidung für oder gegen die Eröffnung des Warzen. fortsatzes noch nicht gefallen ist, diese Symptome trotzdem manchmal den Ausschlag zugunsten einer Aufmeißelung geben könnten und auch oft gegeben haben. Und dies mit Unrecht, denn das Erkennen der zugrunde liegenden Myalgien und ihre zweckentsprechende Behandlung hätten mit Sicherheit den Eingriff verhütet. Ich habe zwar nie einen auf Grund der eben geschilderten Indikation operierten Fall gesehen; wohl aber sah Halle ihrer eine große Anzahl. Die Schmerzen waren nach der Operation auf 2-3 Monate gebessert, traten aber dann in gieicher oder größerer Intensität wieder auf. Es muß daher mit allem Nachdruck auf diese Genese der Symptome hingewiesen werden.

3. Bisher konnte man noch bei den eben erwähnten Symptomen mit einer gewissen Berechtigung den Standpunkt verfechten, daß sie allein wohl kaum die Indikation zur Aufmeißelung abgeben könnten. Anders wird dies jedoch in jenen Fällen, wo bei bestehender eitriger Erkrankung der Nebenhöhlen, speziell der Stirnhöhle und des Siebbeines, plötzlich intensive Stirnkopfschmerzen, Druckschmerzhaftigkeit des Nervus Supra- und Infraorbitalis, Brechreiz, Schwindel usw. auftreten. Hier können die aufgezählten Erscheinungen ohne Rücksichtnahme auf etwa bestehende. Myalgien noch viel leichter als beim Ohre die Ursache zu oft entstellenden radikalen Eröfnungen der erkrankten Nebenhöhlen bilden. Derartiger Fälle konnte ich bei Halle eine ganze Reihe sehen. Hier wird der Operateur erst längere Zeit nach dem Eingriff, wenn die anfangs verschwundenen Kopfschmerzen nach einigen Wochen wieder auftreten, darauf aufmerksam, daß vielleicht doch eine andere Affektion diesen Beschwerden zugrunde liege. Mit Recht weist Halle gegenüber Winkler (7) auf diesen wichtigen Punkt hin und weitere Erfahrungen bestätigen diese Ansicht vollkommen. Oft gelingt es, durch rechtzeitige Erkennung der Myalgien 
die Operation zu vermeiden, wie z. B. folgende Krankengeschichte zeigt:

Ein 35 jähriger Fachkollege klagt seit Jahren über heftige Kopfschmerzen und steht deshalb immer wieder bei Oto-Rhinologen und Nervenärzten in Behandlung. Oberkieferhöhlen anscheinnd gesund, in der Nase soll einmal Eiter gesehen worden sein. Wegen der Stirnkopfschmerzen, die sich speziell bei Druck auf die typischen Schmerzstellen steigern, wird eine Stirnhöhleneiterung bei verschlossenem Ausführungsgang angenommen und die Operation von anderen empfohlen.

Bei neuerlicher Untersuchung (Halle) in der Nase etwas schleimiges Sekret, kein Eiter an typischer Stelle. Spülung beider Oberkieferhöhlen, Kontrolle des Siebbeins sowie Bougierung und Spülung der Stirnhöhlen ohne pathologischen Befund. - Sehr starke Myalgien, an die der Kollege nicht glauben will, die sich jedoch nach 8 tägiger Behandlung bessern. Völliges Wohlbefinden nach 4 Wochen.

Nicht immer aber gelingt es, den Patienten durch rechtzeitige Diagnosenstellung vor einer Operation zu bewahren. So konnte ich bei Halle u. a. eine ca. 6ojährige Patientin sehen, die wegen des Syndromes: chronische Stirnhöhleneiterung - hochgradige Stirnkopfschmerzen nicht weniger als $6 \mathrm{mal}$ extra- und intranasal von hervorragenden Klinikern operiert worden war, ohne jeden bleibenden Erfolg (die Beschwerden stellten sich nach mehrwöchentlicher Pause mit unverminderter Heftigkeit wieder ein), dagegen mit auffallender kosmetischer Entstellung. Erst die Behandlung der gleichzeitig bestehenden Myalgien brachte in wenigen Wochen Heilung. Diese Fälle ließen sich, wenn man nach ihnen forscht, zu Dutzenden aufzählen und beweisen nur immer wieder, wie wenig Beachtung der beherzigenswerte Hinweis Halles gefunden hat.

Während die Behandlung nach Halle-Peritz bei typischen Myalgiefällen gewöhnlich in verhältnismäBig kurzer Zeit zum Ziele führt, konnte ich auch sichere Myalgien sehen, bei denen diese Behandlung anscheinend machtlos war. So behandelte ich eine ca. 3ojährige Frau mit schwersten Myalgien, die 8 Tage hindurch täglich zweimal massiert wurde - ohne jede Besserung; im Gegenteil, die Beschwerden nahmen eher noch zu. Dann blieb Patientin plötzlich aus. Einige Zeit später erfuhr ich von ihrem Gatten, daß sie damals an einem schweren inneren Leiden erkrankte, dessen Natur er nicht angeben konnte, und damit wochenlang liegen mußte. Es ist auf Grund dieser und ähnlicher Krankengeschichten die Annahme nicht ganz von der Hand zu weisen, daß die Myalgien in solchen Fällen entweder Prodromal-oder sogar schon Begleitsymptome einer inneren Erkrankung sein können, was natürlich das Versagen der üblichen Behandlung zur Genüge erklären 
würde. Jedenfalls muß man auf solche gegenüber der Behandlung refraktäre Fälle achten und sie am besten rasch dem Internisten zuweisen.

Es erübrigt noch, eine Erklärung für das Zustandekommen der so verschiedenartigen Symptome der Myalgien zu geben oder sie wenigstens zu versuchen. Halle-Peritz fassen die Schmerzen einerseits als Folge des Druckes der erkrankten Muskeln auf die darunterliegenden Nerven (n. auricularis vagi) oder andererseits bedingt durch Zerrung derselben infolge Zusammenziehung der Galea aponeurotica auf. Durch letzteren Modus würden besonders die Trigeminusäste betroffen. Nun sind aber die bei dieser Annahme notwendigen anatomischen Beziehungen zwischen Sternocleidomastoideus und Cucullaris auf der einen Seite und der Kopfschwarte auf der anderen Seite keineswegs regelmäßige oder innige, indem ein Ausstrahlen der Sehnen in die Kopfșchwarte nur relativ selten angetroffen wird. Es dürfte daher die Erklärung PeritzHalles nur für einen Teil der Fälle zutreffen. Für die übrigen Fälle können wir aber einen anderen, ganz plausiblen Modus als Entstehungsursache annehmen, der gleichzeitig auch die auffallende Tatsache erklären könnte, warum gerade die beiden genannten Muskeln gewöhnlich fast isoliert zu erkranken pflegen. Während Halle z. B. zur Aufklärung dieses Punktes mit Recht auf die Kälteinsulte hinweist, die speziell nachts gerade diese beiden Muskeln treffen, oder Rosenbach die unnatürliche, gezwungene Kopfhaltung im festen Schlafe dafür verantworlich macht, möchte ich nur daran erinnern, daß diese beiden Muskeln sich auch entwicklungsgeschichtlich wesentlich von den übrigen Muskeln unterscheiden, was ganz leicht eine gesteigerte Vulnerabilität derselben zur Folge haben könnte. Außerdem werden ja beide Muskeln vom N. accessorius innerviert, der schon durch sèinen alten Namen: accessorius ad vagum seine nahe Verwandtschaft zum $N$. vagus und dessen Ästen zu erkennen gibt. Man kann daher auch ebensogut annehmen, daß die Auslösung der Schmerzempfindung übet. das Zerebrum geschieht, etwa durch Überspringen des Reizes vom Akzessoriuskern auf die benachbarten Kerne, speziell des Vagus nnd des Trigeminus. Dafür sprechen auch einzelne bei Myalgien vorkommende Sensationen wie z. B. Schwirdel, Ohrensausen, die man nicht gut, wie Peritz es tut, ,durch verschiedene am Trommelfell auftretende abnorme Spannungen" erklären kann, sondern die wohl nur zentral bedingt sein dürften.

In kurzer Zusammenfassung meiner Ausführungen läßt sich etwa folgendes sagen:

I. Die Myalgien des Sternocleidomastoideus und Cucullaris sind nicht nur häufig, sondern auch ein für den Facharzt überaus wichtiges Krankheitsbild, bei dessen Kenntnis und Beachtung manche sonst leichtmögliche Fehldiagnose vermieden wird. 
2. Die richtige Diagnose und zweckgemäße Behandlung der Myalgien wird Patienten und Arzt in gleicher Weise durch überraschende Erfolge befriedigen.

3. Die Entstehung der so verschiedenen Symptome bei Myalgien kann in vielen Fällen in den engen örtlichen anatomischen Beziehungen gelegen sein, in anderen Fällen aber muß man wohl eine zentrale Ursache annehmen.

\section{Literatur.}

I. Halle: Monatsschrift f. Ohrenheilk. Y9I I, Bd. 45, S. 766 .

- Arch. f. Laryng., Bd. 29, H. I.

2. Peritz: Med. Klinik 1906, Nr. 44-46.

3. Bárány: Verhandlg. d. deutsch. otol. Ges. I9r2, S. Irg.

4. Rosenbach: D. med. Wochenschr. I886, Nr. I2-13.

5. Brühl: Berl. laryng. Ges., 23. II. I912.

6. Claus: Ibidem.

7. Winckler: Arch. f. Laryng., Bd. 29, H. I.

8. Grahe: Med. Klin. I92I, Nr. 29. 